Salomon Katembera Ciza ${ }^{1}$

Jean-Fiston MIKWA ${ }^{2}$

Augustin Cirhuza MaleKezi ${ }^{3}$

Valéry GOND ${ }^{4}$

Faustin BOYEMBA BOSELA 5

${ }^{1}$ Faculté des sciences et des sciences appliquées Université officielle de Bukavu BP 570, Bukavu

République démocratique du Congo

2 Faculté des sciences agronomiques Université de Kisangani

BP 2012, Kisangani

République démocratique du Congo

${ }^{3}$ Institut supérieur des techniques médicales de Bukavu

République démocratique du Congo

${ }^{4}$ Cirad

UPR BSEF

Campus international de Baillarguet 34398 Montpellier Cedex 5

France

${ }^{5}$ Faculté des sciences Université de Kisangani BP 2012, Kisangani

République démocratique du Congo

\section{Identification des moteurs de déforestation dans la région d'Isangi, République démocratique du Congo}

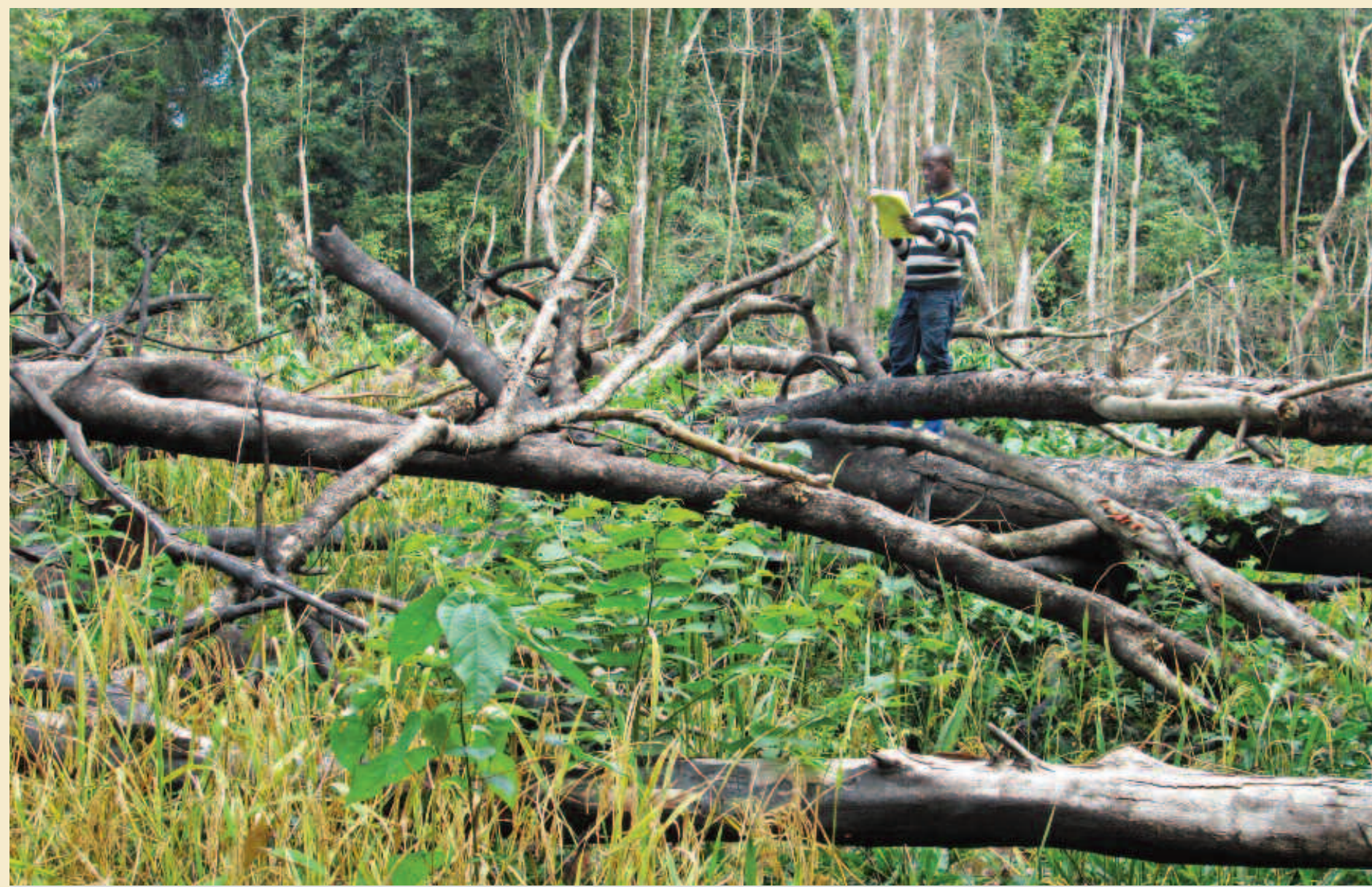

Photo 1.

Coupe et brûlis de la couverture forestière permettant les productions agricoles (riz, maïs et manioc). Photo K. Ciza. 


\section{RÉSUMÉ}

\section{IDENTIFICATION DES MOTEURS \\ DE DÉFORESTATION DANS LA RÉGION D’ISANGI, RÉPUBLIQUE DÉMOCRATIQUE DU CONGO}

La présente étude analyse la perte du couvert forestier dans la zone du projet pilote REDD+ intégré d'Isangi entre 2002 et 2010. La région est l'une des zones de la République démocratique du Congo où les ressources naturelles sont soumises à une forte pression anthropique. Cette étude a permis, grâce aux techniques de détections multi-temporelles des changements combinées aux enquêtes menées sur le terrain, de cartographier les différentes classes d'occupation des sols mais aussi de déterminer les zones les plus affectées par la perte du couvert forestier. Le taux annuel de déforestation est évalué à 0,13\% (330 ha par an) et les émissions associées représentent environ 196000 tonnes de $\mathrm{CO}_{2}$ par an. Les résultats des entretiens auprès des villageois indiquent que les principales causes de la déforestation et de la dégradation des forêts sont l'agriculture itinérante sur brûlis et l'exploitation de bois (bois de chauffage, charbon de bois et bois de construction). Les préconisations afin de ralentir le phénomène de déforestation dans la région sont d'améliorer la production agricole, de formaliser l'exploitation de bois et de diversifier les sources d'approvisionnement des produits ligneux.

Mots-clés : taux de déforestation, émissions de $\mathrm{CO}_{2}$, REDD+, télédétection, causes de déforestation, occupation des sols, République démocratique du Congo.

\section{ABSTRACT}

\section{IDENTIFYING THE DRIVERS OF DEFORESTATION IN THE ISANGI REGION, DEMOCRATIC REPUBLIC OF CONGO}

This study analyses the loss of forest cover in the Isangi integrated REDD+ pilot project zone from 2002 to 2010 . This is one of the regions in the DRC where human pressures are most severe. The study used multi-temporal remote sensing of changes in forest cover combined with field surveys to map the different land-use classes and to determine the zones most severely affected by forest cover losses. The rate of deforestation is estimated at $0.13 \%$ per year (330 ha) and the associated emissions at $196000 \mathrm{t}$ $\mathrm{CO}_{2}$ per year. The results of the surveys conducted among the villagers indicate that the main causes of deforestation and forest degradation are slash-andburn farming and logging (for firewood, charcoal and construction timber). The recommendations made to slow deforestation in this region are to improve agricultural production, bring logging into the formal sector and diversify the sources of supply of timber products.

Keywords: deforestation rate, $\mathrm{CO}_{2}$ emissions, REDD+, remote sensing, causes of deforestation, land use, Democratic Republic of Congo.

\section{RESUMEN}

\section{IDENTIFICACIÓN DE LOS MOTORES DE DEFORESTACIÓN EN LA REGIÓN DE ISANGI, REPÚBLICA DEMOCRÁTICA DEL CONGO}

El presente estudio analiza la pérdida de cubierta forestal en la zona del proyecto piloto REDD+ integrado de Isangi entre 2002 y 2010 . Esta es una de las zonas de la República Democrática del Congo cuyos recursos naturales están sometidos a una fuerte presión antrópica. Este estudio ha permitido, gracias a análisis multitemporales de cambios combinados con encuestas de campo, cartografiar los diferentes tipos de uso del suelo y determinar también las zonas más afectadas por la pérdida de la cubierta forestal. La tasa anual de deforestación se evaluó en $0.13 \%$ (330 ha por año) y las emisiones asociadas representan aproximadamente 196000 t de $\mathrm{CO}_{2}$ emitidas al año. Los resultados de las entrevistas mantenidas con los campesinos indican que las principales causas de la deforestación y la degradación forestal son la agricultura itinerante de roza y quema y la explotación maderera (leña, carbón de leña y madera de construcción). Las recomendaciones para frenar el fenómeno de deforestación en la región son: mejorar la producción agrícola, formalizar el aprovechamiento maderero y diversificar las fuentes de suministro de productos madereros.

Palabras clave: tasa de deforestación, emisiones de $\mathrm{CO}_{2}$, REDD+, teledetección, causas de la deforestación, uso del suelo, República Democrática del Congo. 


\section{Introduction}

La déforestation représente $17,4 \%$ des émissions mondiales de $\mathrm{CO}_{2}$ (GIEC, 2007). Pour réduire les émissions liées à cette déforestation, plusieurs mécanismes ont été envisagés comme le mécanisme REDD+ (réduction des gaz à effet de serre, GES, dus à la déforestation et à la dégradation forestière). Les pays comme la République démocratique du Congo (RDC), concernés par des accords internationaux visant à lutter contre le changement climatique, sont encouragés à développer des « projets pilotes». Ces projets doivent nourrir les négociations afin de déterminer des règles facilitant la mise en place du mécanisme REDD+. La RDC dispose de 145 millions d'hectares de forêts tropicales humides (FAO, 2010). Ces forêts subissent depuis plusieurs décennies une forte pression anthropique. Le taux de déforestation en RDC est estimé à 1,2\% par an pour la période 2005-2010 (OFAC, 2012). Cette déforestation varie spatialement et reste corrélée à la densité et aux activités de la population (OFAC, 2012). Parmi ces dernières, l'agriculture, le pâturage et la récolte de bois énergie sont les principales causes de la déforestation (GTCR, 2012 ; Geist et Lambin, 2001). Pour Mayaux et al., (2003), la déforestation en Afrique centrale résulte principalement de l'agriculture itinérante sur brûlis. Or, ce type d'agriculture est considéré comme durable dans le respect de son caractère itinérant et de sa longue durée de jachère (Dounias, 2000).

Dans ce contexte, l'un des projets initiés par la RDC est le Projet pilote REDD+ géographiquement intégré d'Isangi (PPRGII). L'objectif de ce projet est de déterminer les causes de la déforestation et de la dégradation forestière. Isangi est un territoire à forte croissance démographique où l'agriculture itinérante sur brûlis constitue la principale activité de subsistance pour les communautés locales. Sous la pression démographique, le système agricole pratiqué n'est plus durable à cause de la généralisation de jachères courtes (2 à 3 ans). On y exerce aussi l'exploitation forestière de bois d'œuvre (exploitation industrielle et artisanale) et la chasse. Ces transformations ont entraîné des changements dans l'utilisation des terres dont les conséquences sont la perturbation du calendrier agricole, la prolifération des ravageurs et des maladies (Bogaert et Mahamane, 2005 ; Turner, 1989).

L'ampleur des changements d'occupation du sol et la connaissance des mécanismes de déforestation sont mal documentées dans la région. C'est pourquoi cette étude vise, dans le cadre du REDD+, à mesurer par satellite les surfaces déforestées et à documenter, par des entretiens avec la population, les mécanismes de cette déforestation.

\section{Matériel et méthodes}

\author{
Site d’étude
}

La zone d'étude a une superficie de 324095 ha. Elle est située à $125 \mathrm{~km}$ à l'ouest de la ville de Kisangani, dans la province orientale de la RDC. Elle est parcourue par un réseau hydrographique dense et est traversée dans sa partie nord par le fleuve Congo et à l'est par la rivière Lomami (figure 1). La végétation correspond à la forêt dense ombrophile et sempervirente, caractéristique du domaine forestier central de la cuvette congolaise. Elle jouit d'un climat équatorial du type Af de la classification de Koppen. Ce climat est caractérisé par des pluies abondantes interrompues par deux saisons sèches respectivement en janvier-février (grande saison sèche) et en juin-août (petite saison sèche). Les fluctuations pluvio-thermométriques sont cependant importantes. Elles varient entre $1500-2000 \mathrm{~mm}$ (moyenne de $1750 \mathrm{~mm}$ ) de pluies et 20 à $30^{\circ} \mathrm{C}$ (moyenne de $25^{\circ} \mathrm{C}$ ) (www.worldclim.org). Les secteurs habités se situent le long des voies de communication (fleuve et route).

\section{Échantillonnage en forêt}

Des missions de terrain ont permis d'identifier les éléments du paysage localisés par GPS (Ozswald et al., 2011). Une fiche de terrain a été élaborée pour décrire chaque point échantillonné et documenté par une série de photos (Ozswald et al., 2012). La compilation de ces fiches a permis d'élaborer une typologie de terrain (forêt, jachère, agriculture et eau). La forêt a été définie en trois différents types : forêts de bas-fond, forêts claires, forêts denses. Pour

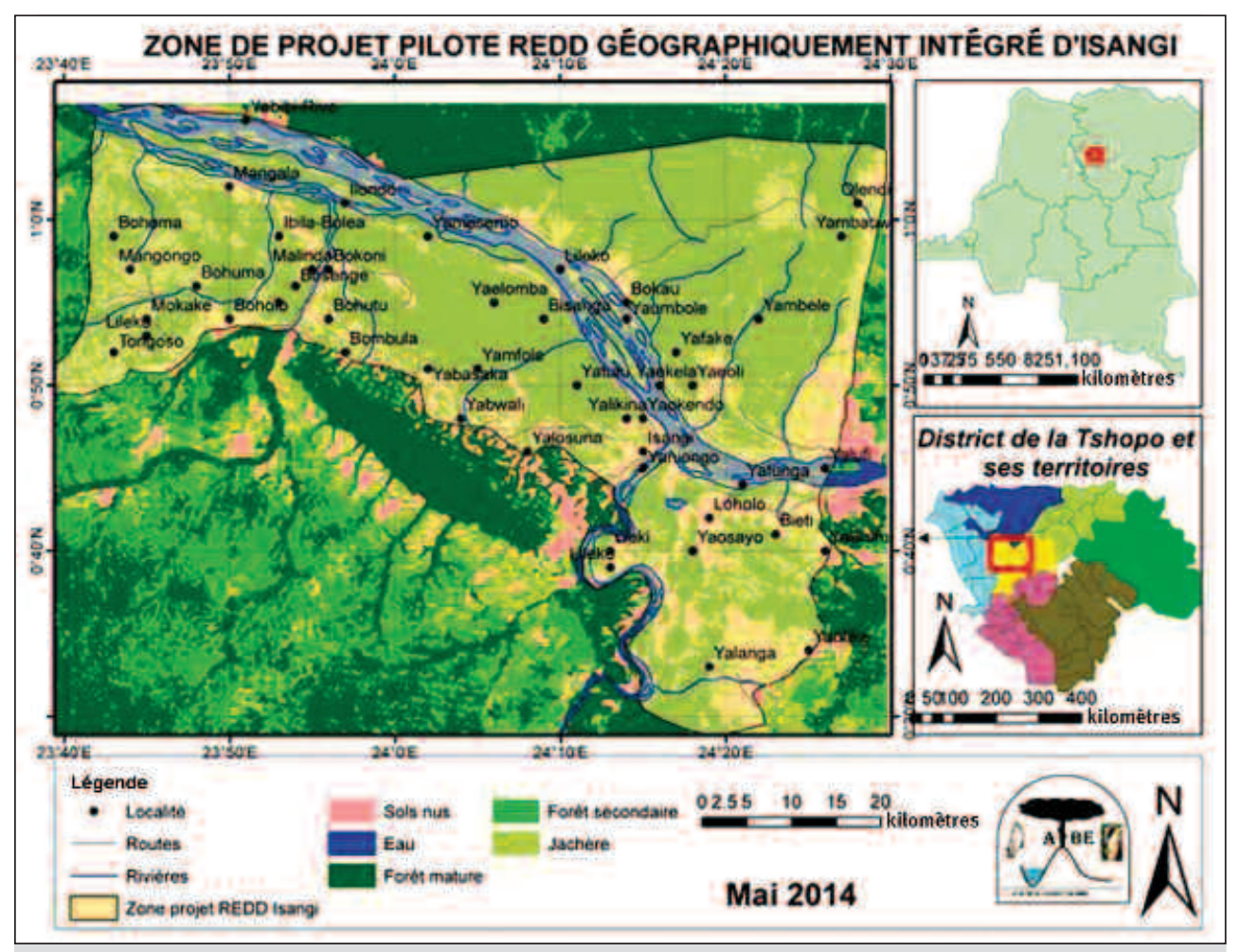

Figure 1.

La zone d'étude et sa localisation par rapport au district de la Tshopo et à la République démocratique du Congo. 
chacun de ces types forestiers, l'intensité de dégradation a été qualifiée en forêts brûlées ou forêts exploitées. La jachère a été catégorisée en trois différents types : moins de 5 ans, entre 5 et 10 ans, plus de 10 ans.

\section{Données satellitaires}

Afin de déterminer la dynamique du couvert forestier, deux images Landsat-7 ETM+ de mars 2002 et février 2010 ont été utilisées. Les bandes spectrales du moyen infrarouge $[1,55-1,75 \mu \mathrm{m}]$, proche infrarouge $[0,78-0,9 \mu \mathrm{m}]$ et rouge $[0,63-0,69 \mu \mathrm{m}]$ ont été exploitées. Les traitements ont été effectués à l'aide des logiciels ArcGIS 1.10, Envi 4.6 et QGIS 1.8. Le prétraitement des deux images a consisté à contrôler leur géopositionnement à l'aide des points de contrôle identifiés sur le terrain (ponts, croisements de chemins, bords de rivière). Les écarts observés étant inférieurs à la taille du pixel (30 m), aucune correction supplémentaire n'a été réalisée. L'analyse par photo-interprétation de l'image 2010 a permis de choisir les zones d'échantillonnage de terrain et de déterminer les zones d'entraînement de la classification supervisée (Ozswald et al., 2012). Une partie des zones d'entraînement (15\%) a été utilisée pour valider la classification (figure 2). L'algorithme de classification retenu est la méthode du maximum de vraisemblance. Il affecte chaque pixel à la classe à laquelle il a la plus grande probabilité d'appartenir. Nous avons utilisé 15 zones d'entraînement pour chaque classe d'occupation du sol identifiée sur les images satellitaires (forêt mature, forêt secondaire, jachère, complexe habitat-agriculture et eau).
L'image 2002 a été classifiée de manière rétrospective, à partir de la ressemblance spectrale des classes de 2010. La validation statistique a été déterminée par la matrice de confusion et l'indice de Kappa (Ozswald et al., 2011). La classification de 2010 a une précision globale de 79,8\% avec un coefficient Kappa de $71 \%$. La classification de 2002 confrontée au manque de données de validation n'a qu'une précision globale de $67,8 \%$ et un indice de Kappa de $64,3 \%$. Nous avons utilisé des données collectées en 2013 au sein de paysages homogènes considérés comme stables durant ces dix dernières années.

\section{Calcul du taux de déforestation}

L'équation 1 a permis de calculer le taux de déforestation pour la période 2002-2010 (Caloz et Collet, 2001). Le taux annuel de déforestation a été obtenu en divisant le taux de déforestation par le nombre d'années d'étude (8 ans) :

$\mathrm{T}_{\text {déforestation }}(\%)=\frac{(\mathrm{S} 2-\mathrm{S} 1)}{\mathrm{S}} \times 100($ équation 1$)$

où :

$\mathrm{S}_{2}$ est l'étendue occupée par la forêt (mature ou secondaire) en 2010 ;

$\mathrm{S}_{1}$ est l'étendue occupée par la forêt (mature ou secondaire) en 2002 ;

S est la superficie occupée par la forêt (mature et secondaire) en 2002.

Les émissions de gaz à effet de serre associées à la déforestation ont été calculées avec l'équation 2 (Mayaux et Achard, 2010) :

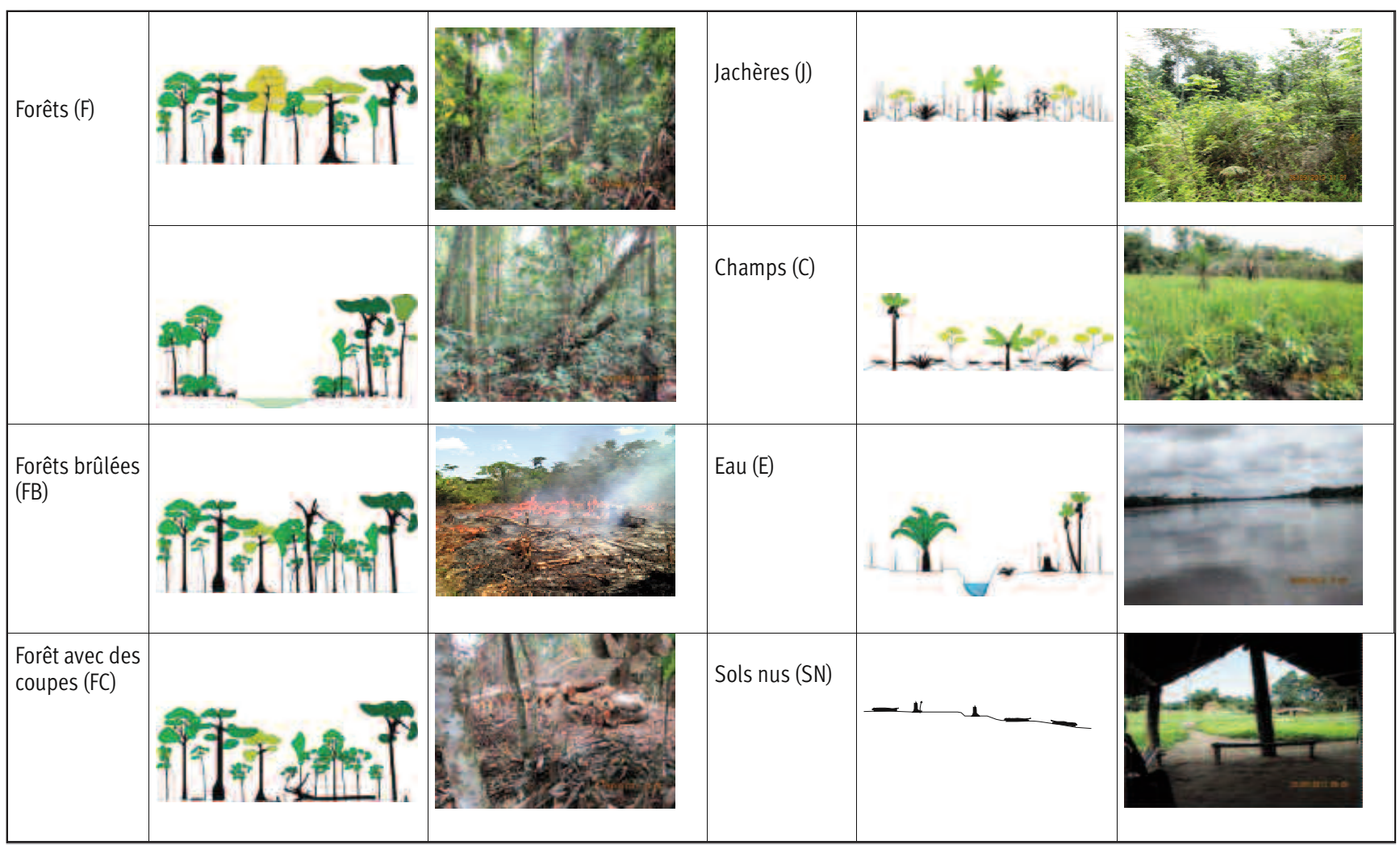

Figure 2 .

Typologie des éléments paysagers caractérisée à partir de l'analyse des images satellitaires entre 2002 et 2010. 


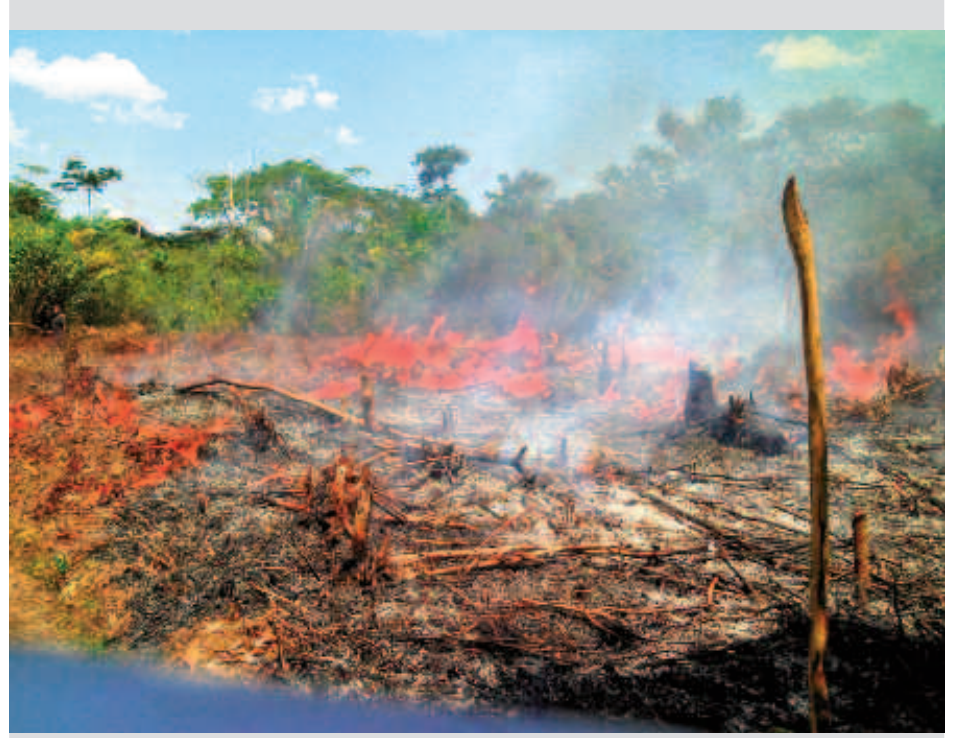

Photo 2.

Pratique d'agriculture itinérante sur brûlis.

Photo K. Ciza.

Émission $=A \times F E$ (équation 2)

Où :

A représente la superficie concernée par la déforestation ; FE représente le facteur d'émissions ou la quantité de gaz perdue par unité de surface.

La région d'Isangi manque d'informations sur le stock de carbone à l'hectare. Pour constituer FE, nous avons eu recours aux valeurs de stock de carbone des zones voisines. À Yangambi (20 km à l'est), le stock de carbone moyen dans la biomasse aérienne de la forêt dense est estimé à 162 tonnes par hectare (Kearsley et al., 2013). Étant donné qu'une tonne de carbone correspond à 3,67 tonnes de $\mathrm{CO}_{2}$, le $F E$ est alors de 594,5 tonnes de $\mathrm{CO}_{2}$ émises à l'hectare.

\section{Entretiens villageois}

Des entretiens ont été menés de juillet à août 2013 dans cinq villages afin d'évaluer la perception de la déforestation par les agriculteurs (annexe I). La carte des changements d'occupation du sol a permis de sélectionner les villages affichant les plus fortes intensités de pression anthropique. Un total de 45 personnes par village (4,3\% de la population) équitablement réparties entre hommes et femmes ont été interrogées. Chaque personne représente une exploitation agricole. Les personnes ayant au moins 25 ans et 10 ans d'ancienneté dans le village ont été sélectionnées pour les entretiens. Chaque exploitation agricole a été définie en fonction de variables agricoles (usage du sol, type d'agriculture, durée de jachère) et forestières (bois énergie, bois de construction). Dans un second temps, l'entretien a abordé la perception des causes de la déforestation (Kristensen et al., 2003). Chaque personne a précisé le type de changement qu'elle a constaté dans la forêt (détérioration, stabilité, accroissement).

\section{Résultats}

\section{Occupation du sol}

Deux cartes ont été produites montrant l'occupation du sol en 2002 et en 2010 (figures 3 et 4). En 2002, les classes forêt mature (192 572 ha) et forêt secondaire (59 004 ha) étaient les classes dominantes. En 2010, seule la forêt mature (202 259 ha) devenait la classe dominante. En 2010, la jachère de 2002 (18 788 ha) a été convertie en complexe habitat-agriculture (28 768 ha) et en forêt secondaire (46 675 ha). La classe complexe habitat-agriculture, densément peuplée, apparaissait dispersée sous forme de petites taches sur l'ensemble de la zone d'étude (tableau I).

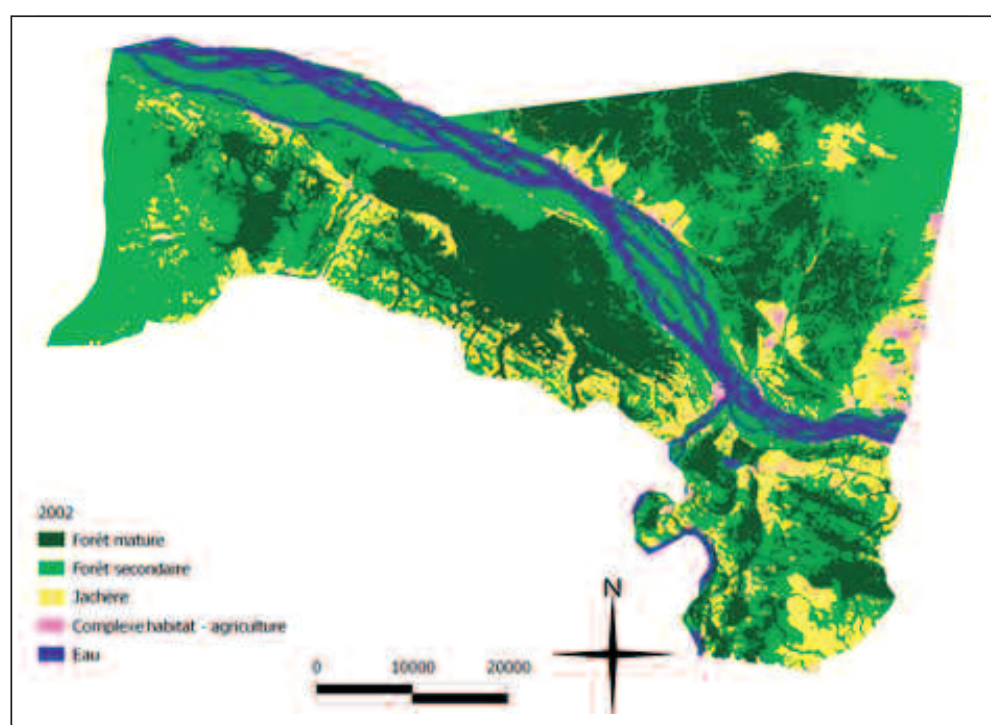

Figure 3.

Classes d'occupation du sol en 2002.

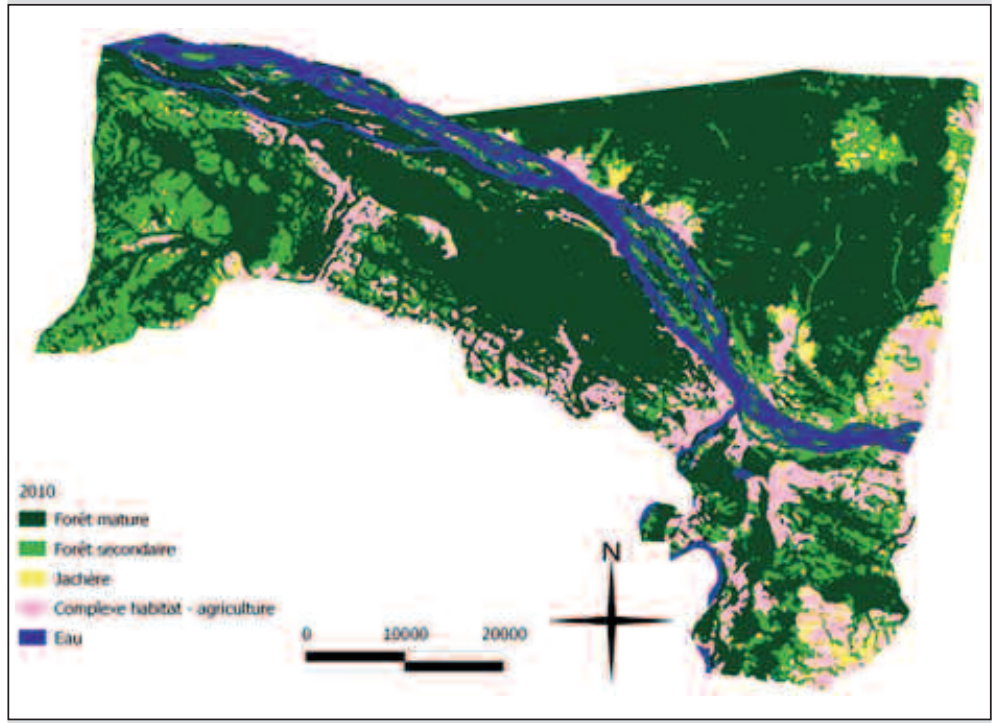

Figure 4.

Classes d'occupation du sol en 2010. 
Tableau I.

Superficie de chaque classe d'occupation du sol.

\begin{tabular}{l|c|c|}
\hline Classe & Surface en $\mathbf{2 0 0 2}($ ha) & Surface en $\mathbf{2 0 1 0}$ (ha) \\
\hline Forêt mature & 192572 & 202259 \\
\hline Forêt secondaire & 59004 & 46675 \\
\hline Jachère & 18788 & 15852 \\
\hline Habitat-agriculture & 23191 & 28768 \\
\hline
\end{tabular}

de la déforestation. La superficie du complexe habitat-agriculture a augmenté de 5577 ha entre 2002 et 2010 , soulignant la pression exercée par la démographie et l'augmentation de la demande de produits agricoles (Megevand et al., 2013). Pour Karsenty (2012), quand la population dépasse 10-15 habitants $/ \mathrm{km}^{2}$, les temps de jachère diminuent forcément et la fertilité décroît. À Isangi la densité est de 12-17 habitants $/ \mathrm{km}^{2}$ (www.afripop.com). L'agriculture dans la région est caractérisée par l'usage de variétés non améliorées et une mauvaise

Dynamique de déforestation

La perte du couvert forestier entre 2002 et 2010 est uniquement observée dans la forêt secondaire. La superficie de la forêt mature a progressé d'environ 4 \% (tableau II). De manière générale, la perte nette du couvert forestier est évaluée à 2642 ha. Cela correspond à un taux de déforestation de 1,05\% pour la période 2002-2010. Le taux annuel de la déforestation est alors estimé à 0,13\%, soit 330 ha de forêt perdus par an. La combinaison des informations relevant de la déforestation et de la quantité de biomasse stockée a permis d'estimer les émissions de $\mathrm{CO}_{2}$ dues à cette déforestation. Les émissions liées à la déforestation sont de 196334 tonnes de $\mathrm{CO}_{2}$ par an.

\section{Les perceptions villageoises}

L'ensemble des personnes interrogées partageaient la même perception de la détérioration du couvert forestier. Trois types d'usage en cause ont été identifiés : l'agriculture itinérante sur brûlis (55,3 \% des personnes interrogées), la production de charbon de bois (27,5\%) et l'exploitation artisanale de bois (16,1\%). Les autres activités paysannes comme l'élevage, la pêche et la chasse ont été rarement citées.

\section{Discussion}

Les classifications d'images satellitaires de 2002 et 2010 ont permis d'identifier les changements d'occupation du sol dans la zone forestière d'Isangi. Le taux de déforestation observé est estimé à 0,13 \% par an. Ce taux est légèrement inférieur à la moyenne nationale qui oscille entre 0,2 et $0,3 \%$ ces vingt dernières années (GTCR, 2012 ; OFAC, 2012). Les activités de subsistance sont la principale cause gestion de la fertilité du sol (Mpoyi et al., 2013). Dans ce contexte de faible productivité, la conversion des terres forestières à l'agriculture s'accélère et la durée de jachère diminue. La durée d'exploitation d'un champ varie entre une (riz, maïs) et deux années (manioc). L'agriculture itinérante sur brûlis traditionnellement pratiquée en RDC s'est maintenue pendant des siècles (Bamba, 2010). Aujourd'hui, le raccourcissement de la durée de jachère impacte le processus de reconstitution forestière (Fournier et al., 2001). À Isangi, les temps de jachère usuels de cinq ans ne concernent plus que $25 \%$ des personnes interrogées (et $8 \%$ à plus de cinq ans) alors que $49 \%$ reconnaissent réduire ce temps de repos à trois ans.

Quant à la surface des forêts secondaires, elle a subi une forte diminution. En plus des activités agricoles, c'est l'exploitation de bois (bois de chauffage, charbon de bois et bois de construction) qui exerce la plus forte pression sur ces forêts. Dans les pays du bassin du Congo, plus de $90 \%$ du volume total de bois récolté sert de bois de chauffage (Marien, 2009). À Isangi, la collecte de bois de chauffage est destinée à la consommation locale et non à l'exportation. Dans les villages, $84 \%$ des personnes interrogées utilisent le bois de chauffage pour la cuisine. Les principaux facteurs qui favorisent son utilisation sont sa capacité à cuire rapidement les aliments et sa disponibilité sur place. La population considère le bois de chauffage comme gratuit et, par conséquent, accessible par tous. Toutefois, l'impact de ce prélèvement reste faible et il est compensé par la régénération naturelle. Le charbon de bois produit à Isangi est exporté vers Kisangani $(90 \mathrm{~km})$. La demande devrait continuer à augmenter dans les prochaines années à cause de la croissance démographique de cette ville (5 à $8 \%$ par an) et de l'évolution des prix de l'énergie (électricité et gaz) (Megevand et al., 2013). Le secteur de la production de charbon de bois est de type traditionnel. Il est caractérisé

Tableau II.

Taux de déforestation entre 2002 et 2010 dans la zone d'étude.

Classe

Forêt mature

Forêt secondaire
Surface en 2002 (ha)

192571

59004
Surface en 2010 (ha)

202259

46675
Taux de déforestation (\%) $+3,8$

$-4,9$




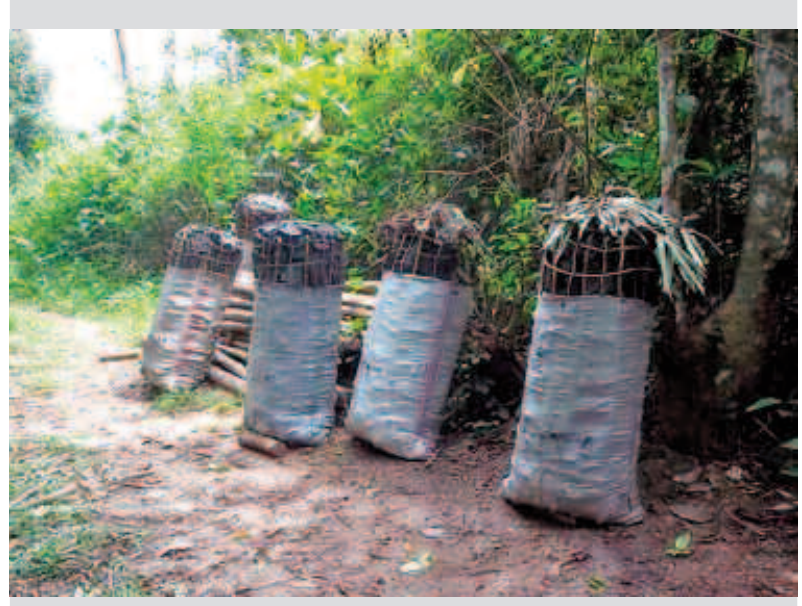

Photo 3.

Sacs de charbon de bois en attente d'être acheminés à Kisangani. Photo K. Ciza.

par une faible efficacité de transformation, une mauvaise organisation de la chaîne de production et l'informalité de la filière. Elle est devenue depuis peu une activité génératrice de revenus pour de nombreux ménages. Dans les villages, 35 personnes (soit $15 \%$ des personnes interrogées) se sont identifiées comme charbonniers. Un charbonnier réalise deux à trois fours par semaine. Aujourd'hui, cette exploitation se pratique en dehors de tout cadre de durabilité et représente une menace certaine pour la forêt.

Le bois est aussi prélevé par la population locale pour la construction des maisons et des accessoires quotidiens (tables, chaises et lits). Ces maisons ont une durée de vie de 5 à 10 ans. Ce sont généralement les forêts secondaires situées à proximité des zones densément peuplées qui sont dégradées.

Dans notre étude, les émissions liées à la déforestation sont estimées à environ 196000 tonnes de $\mathrm{CO}_{2}$. Ce chiffre est à prendre avec précaution car il est issu de mesures réalisées à Yangambi dans des forêts matures (Kearsley et al., 2013). De plus, les principaux impacts de déforestation sur notre site d'étude se cantonnent aux forêts secondaires dont la biomasse est inférieure à celle des forêts matures. Malheureusement, aucune mesure de biomasse dans ce type de forêt n'était à notre disposition.

La situation détaillée dans cette étude montre la nondurabilité du système agricole et forestier d'Isangi. Le modèle agricole pratiqué est responsable d'une perte régulière du couvert forestier. Sous la pression démographique, ce modèle continuera de causer des pertes de superficie forestière. La forêt représente l'enjeu socio-économique de l'avenir des populations d'Isangi (bois de chauffage, charbon de bois et bois de construction). Sa continuelle détérioration aura des conséquences dramatiques pour la population (Marien et al., 2013). Afin d'enrayer le phénomène, il est urgent de développer des actions pour améliorer la gestion forestière. Cette amélioration doit contribuer à ralentir, voire arrêter la déforestation provoquée par l'agriculture itinérante sur brûlis. Pour cela, le développement de programmes d'amélioration de la fertilité des sols, de sédentarisation des exploitants et de soutien aux petits exploitants est nécessaire. Combinés aux politiques et mesures d'accompagnement en matière d'aménagement du territoire, de tels programmes permettraient de produire plus sur la même superficie. Cette intensification agricole inciterait les exploitants à ne pas étendre leurs activités et aiderait à préserver la forêt.
Longtemps ignoré, le secteur de l'exploitation de bois (bois de chauffage, charbon de bois et bois de construction) est maintenant reconnu comme étant un secteur majeur de l'exploitation forestière. À Isangi comme partout en RDC, ce secteur est caractérisé par la non-réglementation, la faible performance et la non-durabilité. Lescuyer et al. (2012) montrent qu'au Cameroun et en RDC la production informelle de bois surpasse déjà la production formelle. Avec l'étalement urbain à Kisangani, la tendance n'est pas susceptible de s'inverser. De nombreux ménages en RDC devraient être équipés en foyers améliorés afin de réduire la consommation de charbon de bois. La lutte contre l'exploitation illégale des forêts passe par la formalisation de la chaîne commerciale de l'exploitation de bois. La mise en place de solutions comme les plantations et l'agroforesterie est à envisager rapidement. D’une manière générale, le développement de ces activités alternatives permettrait de lutter efficacement contre les émissions de $\mathrm{CO}_{2}$ associées à la déforestation et de mettre à disposition des populations locales des ressources en bois non négligeables (Marien et al., 2013).

\section{Conclusion}

Cette étude a été conduite dans la région d'Isangi en République démocratique du Congo. Cette région fait partie des vastes régions des forêts tropicales soumises au processus de déforestation et de dégradation forestière. L'étude a permis, grâce à la télédétection, de mettre en évidence les changements d'occupation du sol entre 2002 et 2010 . Le taux annuel de déforestation y est estimé à $0,13 \%(0,2$ à $0,3 \%$ en RDC) et concerne surtout la forêt secondaire. L'impact des pratiques de la population sur la dynamique forestière a été analysé grâce aux entretiens menés sur le terrain. Les résultats montrent que les causes principales sont les réductions du temps de jachère de l'agriculture sur brûlis et l'exploitation de bois (bois de chauffe, charbon de bois et bois de construction).

En dépit de ce faible taux annuel de déforestation, l'absence d'une politique de gestion forestière susceptible de diminuer l'impact de ces activités anthropiques sur le couvert forestier conduirait à l'augmentation des émissions de $\mathrm{CO}_{2}$. La conservation du paysage forestier de la zone d'Isangi passe par des mesures appropriées et durables tenant compte de l'amélioration de la production agricole, de la formalisation du secteur bois énergie et bois de construction, enfin de la diversification des sources d'approvisionnement de ces produits ligneux.

\section{Remerciements}

Les auteurs remercient l'Union européenne et le Cifor pour avoir financé cette étude. Merci à Johan Oszwald pour nous avoir autorisés à utiliser ses représentations des éléments du paysage (figure 2) ainsi que la Nasa pour avoir mis gracieusement à disposition les images Landsat nécessaires à cette étude. Nous remercions aussi les autorités ainsi que tous les techniciens de terrain du Projet pilote REDD+ géographiquement intégré d'Isangi pour nous avoir permis de mener à bien nos recherches. 


\section{Annexe I.}

\section{Questionnaire d'enquête utilisé pour l'étude.}

Monsieur, Madame, Mademoiselle, Pour nous permettre de bien réaliser nos travaux de recherche portant sur les problèmes de déforestation et de dégradation forestière dans vos villages, nous sollicitons votre collaboration en acceptant de répondre objectivement aux questions ci-dessous.

Étant donné le caractère scientifique du travail, nous vous garantissons l'anonymat. Nous vous demeurons reconnaissants et vous remercions d'avance pour votre contribution.

\section{IDENTIFICATION}

- Nom de la localité :

- Type de famille (monogamique, polygamique) :

- Taille du ménage :

NB : ménage signifie l'ensemble des personnes vivant dans une maison et partageant le même repas.

- Collectivité : groupement : clan

- Âge de l'enquêté :

a. Si moins de 25 ans, arrêter l'enquête

b. Si plus de 25 ans, poursuivre l'enquête

- Sexe de l'enquêté :

- Statut de l'enquêté dans le ménage :

a. Conjoint

b. Conjointe

c. Fils ou fille du ménage

\section{QUESTIONS PRÉLIMINAIRES}

2.1. Depuis combien de temps habitez-vous dans ce village? Si moins de 10 ans, arrêter l'enquête

Si plus de 10 ans, continuer avec l'enquête

2.2. Quelle est votre principale activité?

Ne pas citer les réponses

a. Agriculteur

b. Exploitation de bois

c. Autres à préciser

Si la réponse à 2.2. est $a$, aller à 3.1.

Si la réponse à 2.2. est b, aller à 4.1 .

Si la réponse à 2.2. est c, aller à 5.1.

\section{QUESTIONS SE RAPPORTANT À L'AGRICULTURE}

3.1. À quelle distance se trouve votre champ par rapport à votre village?

3.2. Combien de champs ouvrez-vous par saison de culture?

3.3. Quelle est la taille de votre champ en hectare ?

3.4. Quels sont les différents types de cultures que vous y plantez?

3.5. Quel type d'agriculture pratiquez-vous?

Ne pas citer les réponses

a. Agriculture itinérante sur abattis-brûlis

b. Agriculture itinérante sur abattis-non-brûlis

3.6. Combien de temps prenez-vous pour abandonner un champ et en ouvrir un autre (durée de jachère) ?

3.7. Combien de temps prenez-vous pour récolter ce que vous avez semé (durée de mise en culture) ?

3.8. L'espace que vous réservez pour vos activités champêtres est-il suffisant?
3.9. Si non, que préconisez-vous pour l'utiliser de manière durable pour les générations futures?

Allez à 5

\section{QUESTIONS SE RAPPORTANT À L'EXPLOITATION DE BOIS}

4.1. Quelle distance parcourez-vous pour aller exploiter le bois? 4.2. Quel mode de prélèvement de bois pratiquez-vous?

Citer les réponses

Artisanal

Semi-artisanal

4.3. Dans quel but pratiquez-vous cette exploitation de bois ?

Ne pas citer les réponses

Plusieurs réponses possibles

a. Fabrication de charbon de bois

b. Bois de chauffe

c. Construction de maisons

d. Construction d'autres accessoires quotidiens de la maison

Si la réponse à 4.3. est a, aller à 4.4.

Si la réponse à 4.3. est b, c, ou d, aller à 4.6.

4.4. Combien de four de charbon de bois produisez-vous par semaine?

4.5. Quel volume de bois rond utilisez-vous pour produire un four?

4.6. Y a-t-il des personnes étrangères qui viennent exploiter le bois dans votre village?

4.7. D'où viennent-elles?

4.8. Elles exploitent où sûrement?

4.9. Qui les autorise à exploiter le bois dans votre village?

\section{QUESTIONS RELATIVES À L'ÉVOLUTION DU COUVERT FORESTIER}

5.1. Quelle est votre impression sur l'évolution de la forêt dans votre terroir depuis que vous y habitez jusqu'à ce jour? Ne pas citer les réponses

Une seule réponse possible

- Amélioration

- Maintien

- Détérioration

Si la réponse à 5.1. est a ou b, aller à 6.1.

Si la réponse à 5.1. est c, aller à 5.2.

5.2. Si détérioration il y a, à quoi serait-elle due directement? Citer les réponses

Quatre réponses possibles

a. Installation des plantations à cultures pérennes (palmier à huile, hévéa, etc.)

b. Agriculture itinérante sur abattis-brûlis

c. Élevage (petit et gros bétail)

d. Colonisation des terres par des immigrés

e. Exploitation industrielle de bois d'œuvre

f. Exploitation semi-industrielle de bois d'œuvre

g. Exploitation artisanale de bois

h. Production de charbon de bois

i. Activités de chasse

j. Production de bois de chauffe

k. Transport (routes, voies fluviales, etc.)

l. Caractéristiques des terres, qualité des terres, topographie

5.3. Si détérioration il y a, à quoi serait-elle due indirectement? 


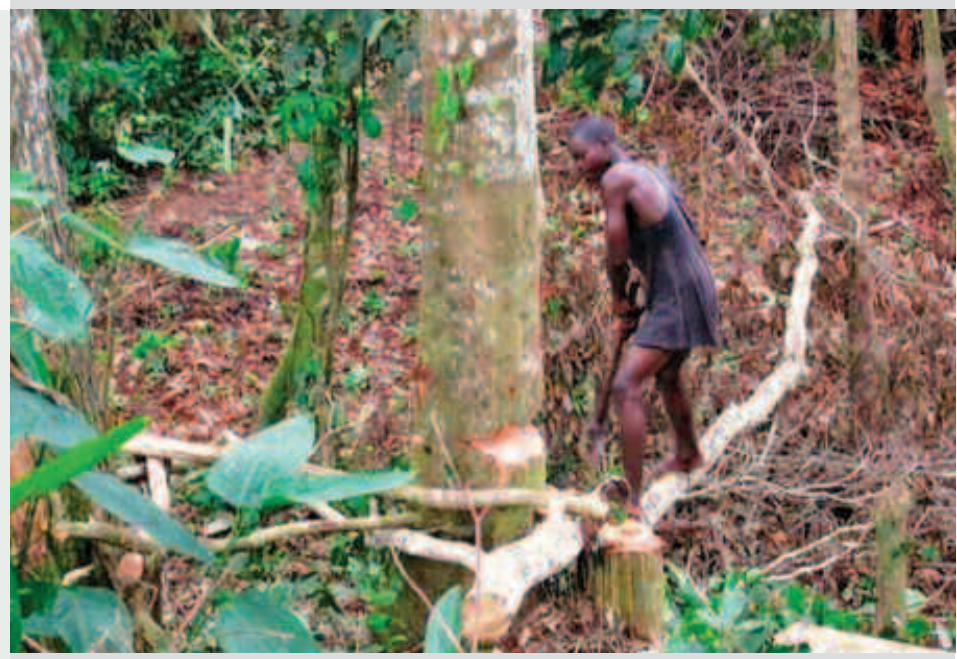

Photo 4.

Exploitation artisanale de bois.

Photo K. Ciza.

Citer les réponses

Quatre réponses possibles

- Augmentation de la densité de la population

- Développement du secteur de marché et de commercialisation

- Urbanisation et industrialisation

- Augmentation des prix, avantages et coûts comparatifs liés aux produits forestiers et agricoles

- Changement agrotechnique

- Déficit administratif

- Attitudes publiques, valeurs et croyances (mentalité sur les limites des forêts)

- Pauvreté

\section{QUESTIONS RELATIVES AU BOIS ÉNERGIE}

6.1. Quel est le type d'énergie que vous utilisez pour la cuisson de votre nourriture?

Ne pas citer les réponses

Plusieurs réponses possibles

- Bois de chauffe

- Charbon de bois

\section{QUESTIONS RELATIVES AUX REVENUS ANNUELS}

7.1. Quelles sont vos différentes sources de revenus dans le ménage (par ordre d'importance) ?

1 . estimation revenu annuel :

$2 .$. estimation revenu annuel :

3. estimation revenu annuel :

4...................................estimation revenu annuel :

\section{Références bibliographiques}

Bamba I., 2010. Anthropisation et dynamique spatio-temporelle de paysages forestiers en République Démocratique du Congo. Thèse, Université libre de Bruxelles, Belgique, $205 \mathrm{p}$.

Bogaert J., Mahamane A., 2005. Écologie du paysage : cibler la configuration et l'échelle spatiale. Annales des Sciences Agronomiques du Bénin, 7 (1) : 39-68.

Caloz R., Collet C., 2001. Précis de télédétection. Traitements numériques d'images de télédétection. Sainte-Foy, Canada, Presses de l'Université du Québec et Agence universitaire de la Francophonie, vol. 3, 386 p.

Devers D., Van de Weghe J. P. (éds), 2007. Les forêts du Bassin du Congo. État des forêts 2006. Partenariat pour les forêts du Bassin du Congo, 256 p. http://carpe.umd.edu/ Documents/2006/LES_FORETS_DU_BASSIN_DU_CONGO_E tat_des_Forets_2006.pdf.

Dounias E., 2000. La diversité des agricultures itinérantes sur brûlis. In : Bahuchet S., De Maret P. (éds). Avenir des peuples des forêts tropicales. 2. Approche thématique. Bruxelles, Belgique, Commission européenne, direction générale VIII, p. 65-106.

Duveiller G., Defourny P., Desclée B., Mayaux P., 2008. Deforestation in Central Africa: Estimates at regional, national and landscape levels by advanced processing of systematically-distributed Landsat extracts. Remote Sensing of Environment, 112: 1969-1981. http://www.sciencedirect.com /science/article/pii/S0034425708000229.

FAO, 2010. Études de cas sur l'évaluation de la dégradation des forêts en RDC. Évaluation des ressources forestières. Rome, Italie, FAO, Document de travail 169, 29 p.

Fournier A., Floret C., Gnahoua G., 2001. Végétation des jachères et succession post-culturale en Afrique tropicale. In : Floret C., Pontanier R. (éds). La jachère en Afrique tropicale. De la jachère naturelle à la jachère améliorée. Le point des connaissances. Montrouge, France, John Libbey Eurotext, vol. 2, 123-168.

GIEC, 2007. Changements climatiques 2007. Rapport de synthèse. Résumé à l'attention des décideurs, 114 p.

GTCR, 2012. Étude qualitative sur les causes de la déforestation et de la dégradation des forêts en République Démocratique du Congo. RDC, GTCR, 165 p.

Karsenty A., 2012. Le rôle de l'agriculture dans la déforestation et la dégradation en RDC. Situation actuelle, perspectives et solutions possibles. Montpellier, France, Cirad, $21 \mathrm{p}$. Kearsley E., de Haulleville T., Hufkens K., Kidimbu A., Toirambe B., Baert G., Huygens D., Kebede Y., Defourny P., Bogaert J., Beeckman H., Steppe K., Boeckx P., Verbeeck H., 2013. Conventional tree height-diameter relationships significantly overestimate aboveground carbon stocks in the Central Congo Basin. Nature Communications, 4. 
Kristensen M., Balslev H., 2003. Perceptions, use and availability of wood plants among the Gourounsi in Burkina Faso. Biodiversity and Conservation, 12: 1715-1739.

Lescuyer G., Cerutti P., Essiane M., Eba’a A., Nasi R., 2012. Évolution du secteur du sciage artisanal dans le bassin du Congo. In : de Wasseige C., de Marcken P., Bayol N., Hiol Hiol F., Mayaux P., Desclée B., Nasi R., Billand A., Defourny P., Eba'a Atyi R. (éds). Les forêts du Bassin du Congo. État des forêts 2010. Luxembourg, Office des publications de l'Union européenne, 97-108.

Marien J.-N., Dubiez E., Louppe D., Larzillière A. (éds), 2013. Quand la ville mange la forêt. Les défis du bois-énergie en Afrique centrale. Versailles, France, Éditions Quæ, 238 p.

Mayaux P., Gond V., Massart M., Pain-Orcet M., Achard F., 2003. Évolution du couvert forestier du bassin du Congo mesurée par télédétection spatiale. Bois et Forêts des Tropiques, 277 : 45-52.

Mayaux P., Achard F., 2010. Overview of carbon and REDD: the needs of MRV systems. REDD-plus' requirements for the Congo Basin Countries? In: Brady M., de Wasseige C. (eds). Monitoring forest carbon stocks and fluxes in the Congo basin, Brazzaville, Republic of Congo, 2-4 February 2010. COMIFAC, 4-7.

Megevand C., Mosnier A., Hourticq J., Klas S., Nina D., Streck C., 2013. Dynamiques de déforestation dans le bassin du Congo : Réconcilier la croissance économique et la protection de la forêt. Washington, DC, USA, Banque mondiale, 201 p. DOI : 10.1596/978-0-8213-9827-2.

Mpoyi M., Nyamwoga B., Kabamba M., Assembe-Mvondo, 2013. Le contexte de la REDD+ en République Démocratique du Congo : Causes, agents et institutions. Bogor, Indonésie, Cifor, Occasional Paper 84, 69 p.

OFAC, 2012. The Forests of the Congo Basin. State of the Forest 2010. Luxembourg, Office des publications de l'Union européenne, 276 p. http://www.observatoire-comifac.net/edf2010.php?l=en.

Oszwald J., Arnauld de Sartre X., Decaëns T., Gond V., Grimaldi M., Lefebvre A., De Araujo Fretas R.L., Lindoso de Souza S., Marichal R., Veiga I., Velasquez E., Lavelle P., 2012. Utilisation de la télédétection et de données socioéconomiques et écologiques pour comprendre l'impact de la dynamique de l'occupation des sols à Pacajà (Brésil). Revue Française de Photogrammétrie et de Télédétection, 198-199: 12-29.

Oszwald J., Gond V., Dolédec S., Lavelle P., 2011. Identification d'indicateurs de changement d'occupation du sol pour le suivi des mosaïques paysagères. Bois et Forêts des Tropiques, 307 (1) : 7-21.

Turner M. G., 1989. Landscape ecology: the effect of pattern on process. Annual Review of Ecology and Systematics, 20: 171-197. 